\title{
Effect of gettered iron on recombination in diffused regions of crystalline silicon wafers
}

\author{
D. Macdonald, ${ }^{a}$ H. Mäckel, and A. Cuevas \\ Department of Engineering, Australian National University, Canberra, ACT 0200, Australia
}

(Received 19 December 2005; accepted 7 February 2006; published online 28 February 2006)

\begin{abstract}
Crystalline silicon wafers, intentionally precontaminated with iron, were diffused with phosphorus and boron, and the recombination properties of the bulk and diffused regions extracted from injection-dependent carrier lifetime measurements. While the phosphorus diffusions were found to getter more than $99 \%$ of the iron from the bulk, the boron diffusions only extracted $65 \%$ in the best case. The presence of this gettered iron caused significant additional recombination in the boron diffused layers, while it had no measurable impact on the phosphorus diffused regions. This may be a consequence of the small capture cross section for holes of interstitial iron. (C) 2006 American Institute of Physics. [DOI: 10.1063/1.2181199]
\end{abstract}

Gettering of metal impurities is an essential step in the production of efficient photovoltaic devices from relatively impure materials, such as multicrystalline silicon. For typical $p$-type bases, this gettering is largely achieved by a front surface phosphorus diffusion, the primary function of which is to create a $p$ - $n$ junction. The most important metal extracted in this way is iron, which can otherwise be responsible for more than one-half of the total recombination events in as-grown multicrystalline material. ${ }^{1}$

Neutron activation analysis of multicrystalline silicon has revealed that large concentrations of iron, and other metals, can be removed by such phosphorus diffusions. In one case, ${ }^{2}$ more than $5 \times 10^{13} \mathrm{~cm}^{-3}$ of Fe was extracted, indicating the removal of both interstitial and precipitated Fe. However, the possible effect of these impurities on the recombination rate in the diffused regions where they eventually reside is rarely considered, despite the fact that the concentration of gettered metals there may be very high. This partly reflects the expected dominance of Auger recombination in these highly doped regions. The purpose of this work is to examine whether realistic concentrations of Fe gettered to both boron- and phosphorus-diffused regions can cause a measurable increase in recombination in those regions, as characterized by the emitter saturation current density $J_{0 E}$. Both $p$ - and $n$-type emitters are of interest, as it is well established that pointlike $\mathrm{Fe}$ has a much greater recombination strength in more lowly doped $p$-type silicon than $n$ type. The gettering efficiency of boron and phosphorus diffusions is also compared.

For this work, we used $220 \mu \mathrm{m}$ thick float-zone wafers with resistivities of $2 \Omega \mathrm{cm}$ ( $n$-type phosphorus doped) and $0.5 \Omega \mathrm{cm}$ ( $p$-type boron doped). Iron contamination was achieved by implanting $70 \mathrm{keV} \mathrm{Fe}^{56}$ to doses of $10^{11}$ or $10^{12} \mathrm{~cm}^{-2}$ through a 30 by $30 \mathrm{~mm}$ silicon aperture. The samples were annealed at $900{ }^{\circ} \mathrm{C}$ for $1 \mathrm{~h}$ in nitrogen gas to distribute the $\mathrm{Fe}$ throughout the wafer thickness. These doses correspond to bulk Fe concentrations of around $4 \times 10^{12}$ and $4 \times 10^{13} \mathrm{~cm}^{-3}$, the latter being similar to the amount of $\mathrm{Fe}$ typically gettered in multicrystalline silicon. Note that these values do not exceed the expected $\mathrm{Fe}$ solubility limit $^{3}$ of

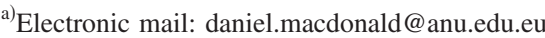

around $5 \times 10^{13} \mathrm{~cm}^{-3}$ at the anneal temperature, meaning little precipitation should occur.

The samples were then subjected to either phosphorus or boron diffusions to generate $p^{+} / n / p^{+}$or $n^{+} / p / n^{+}$structures. Boron diffusions were performed at temperatures of 940 or $990{ }^{\circ} \mathrm{C}$; while for phosphorus, the temperatures were 830 and $870{ }^{\circ} \mathrm{C}$. These conditions created nominally "light" and "heavy" diffusions. After annealing in forming gas at $400{ }^{\circ} \mathrm{C}$ for $40 \mathrm{~min}$ to improve the surface passivation qualities of the phosphorus- or boron-rich glass films which grew during diffusion, the effective lifetimes and emitter saturation currents were measured using the quasi-steady-state photoconductance technique. $^{4-6}$ The sheet resistances of the light and heavy diffusions were approximately 150 and $55 \Omega / \square$ for both the boron and phosphorus diffusions.

Effective lifetime measurements, as a function of excess carrier density $\Delta n$, are shown in Fig. 1 for the lightly borondiffused $n$-type samples. Data for the nonimplanted control and the two Fe-implanted samples are shown. The plot shows that the injection dependence of the measured lifetime can be reconstructed using two components-an injectionindependent bulk lifetime due to Shockley-Read-Hall (SRH) recombination through defects $\tau_{\text {bulk-SRH }}$ (indeed the lack of injection dependence is expected for interstitial Fe in $n$-type silicon), ${ }^{7}$ and a component associated with recombination in the surface emitter regions. This latter component is

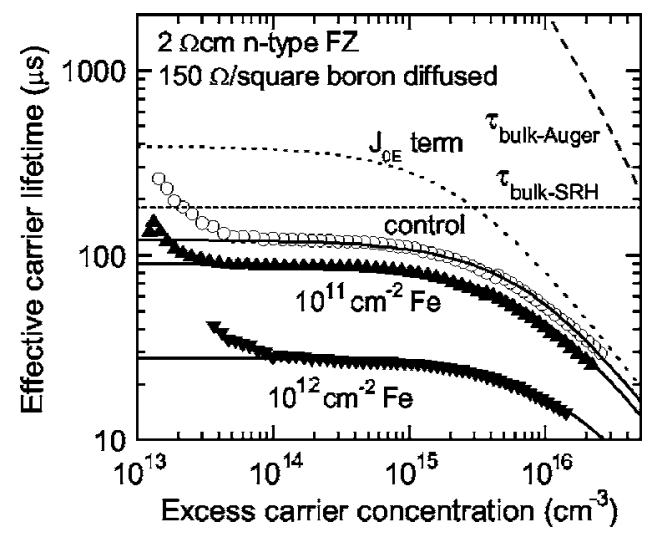

FIG. 1. Effective lifetimes as a function of excess carrier density for the light boron diffusions. The solid lines are fits to determine the bulk lifetime $\left(\tau_{\text {bulk-SRH }}\right)$ and emitter $\left(J_{0 E}\right)$ components. 
TABLE I. Extracted bulk lifetimes at $\Delta n=10^{15} \mathrm{~cm}^{-3}$ for the controls and heaviest $\mathrm{Fe}$ dose, and corresponding values of $\tau_{\mathrm{Fe}},[\mathrm{Fe}]$ in the base, and the percentage of $\mathrm{Fe}$ gettered to the surface diffusions.

\begin{tabular}{lccccc}
\hline \hline $\begin{array}{l}\text { Wafer type, } \\
\text { diffusion }\end{array}$ & $\begin{array}{c}\tau_{\text {bulk-SRH }} \\
\text { control }\end{array}$ & $\begin{array}{c}\tau_{\text {bulk-SRH }} \\
\text { Fe-doped }\end{array}$ & $\begin{array}{c}\tau_{\mathrm{Fe}} \\
{[\mathrm{Fe}]} \\
\left(\mathrm{cm}^{-3}\right)\end{array}$ & $\begin{array}{c}\% \mathrm{Fe} \\
\text { gettered }\end{array}$ \\
\hline$n$-type, B, heavy & $50 \mu \mathrm{s}$ & $57 \mu \mathrm{s}$ & $92 \mu \mathrm{s}$ & $1.4 \times 10^{13}$ & $65 \%$ \\
$n$-type, B, light & 180 & 34 & 42 & $3.1 \times 10^{13}$ & 25 \\
$p$-type, P, heavy & $>15$ & $>13$ & $>50$ & $<2.0 \times 10^{11}$ & $>99$ \\
$p$-type, P, light & 50 & 25 & 50 & $2.1 \times 10^{11}$ & $>99$ \\
\hline \hline
\end{tabular}

characterized by the emitter saturation current density $J_{0 E} \cdot{ }^{6}$ The reduction in lifetime at higher carrier densities in Fig. 1 is due entirely to recombination in the emitter, and allows unambiguous extraction of $J_{0 E}$ (the impact of Auger recombination is negligible, and in any case can be subtracted). The apparent increase in lifetime at excess carrier densities below $10^{14} \mathrm{~cm}^{-3}$ appears to be an artifact caused by modulation of the depletion region width. ${ }^{8}$ Its presence does not significantly affect the extraction of $J_{0 E}$ and $\tau_{\text {bulk-SRH. }}$.

The measured $J_{0 E}$ values were divided by 2 to give the saturation current density per side. This is appropriate when the bulk diffusion length is sufficiently long for lightgenerated carriers to reach both surfaces. This condition is satisfied for most samples here, but in some cases the diffusion length is slightly less than the sample thickness. In these cases, the true values of $J_{0 E}$ could be somewhat larger, although only by a factor significantly less than 2 . Such uncertainties do not affect the main conclusions reached here.

The extracted values of the bulk lifetime allow an estimation of the amount of Fe remaining in the base after diffusion. The lifetime attributable to $\mathrm{Fe}$ in the base is given by $1 / \tau_{\mathrm{Fe}}=1 / \tau_{\text {bulk-SRH }}-1 / \tau_{\text {bulk-SRH-control }}$. The use of the control data subtracts out the impact of other bulk recombination processes such as grown-in or process-induced defects.

Since the impact of a given amount of interstitial Fe on the recombination lifetime in both $n$ and $p$-type silicon is well known (in $n$ type, it is simply in interstitial form, in $p$ type, as FeB pairs prior to illumination), ${ }^{7}$ we may calculate the corresponding $\mathrm{Fe}$ concentration $[\mathrm{Fe}]$ in the base, and thus the gettering efficiency of the diffusions can be determined. For the $p$-type samples, the $[\mathrm{Fe}]$ results were confirmed using the well-established technique based on lifetime measurement before and after splitting the FeB pairs. 9,10

Table I lists the gettering efficiencies estimated in this way, keeping in mind that the diffusion at each surface contains one-half of the gettered Fe. The data shown are for the samples implanted with the higher Fe dose, since in this case the uncertainties were reduced, however, the gettering efficiencies were similar for the lower Fe dose. Note that the gettering efficiency of the phosphorus diffusions is much greater than for boron diffusions, despite the fact that a greater number of boron atoms are required to achieve the same sheet resistance, due to a lower carrier mobility.

Figure 2 shows the $J_{0 E}$ values (per side) extracted for the two boron diffusions. There is a clear two- to three-fold increase in the saturation current for the highest Fe dose. Figure 3 shows the corresponding results for the two phosphorus diffusions. Even though more than $99 \%$ of the Fe is present in the diffused regions, it is striking that there is no measurable impact on the $J_{0 E}$ values.

Downloaded 28 Feb 2006 to 150.203.45.136. Redistribution subject to AlP license or copyright, see http://apl.aip.org/apl/copyright.jsp

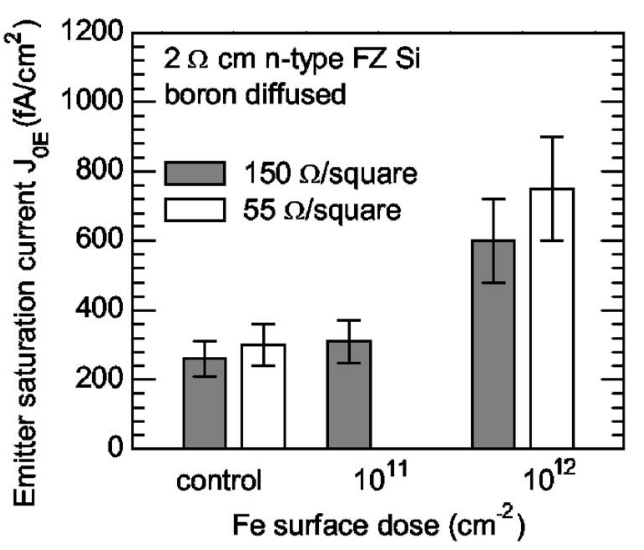

FIG. 2. Emitter saturation currents for the boron diffused samples as a function of $\mathrm{Fe}$ dose.

These observations can be explained by the large differences in capture cross sections for electrons and holes for the likely forms of Fe in these samples. Since the diffused regions are always in low injection, the recombination rate through the Fe-related centers will be determined by the minority carrier capture cross section. In the $n$-type phosphorus diffusions, the Fe is likely to be in interstitial form, and the corresponding cross section (for holes) is very small, ${ }^{3}$ around $7 \times 10^{-17} \mathrm{~cm}^{2}$. For $p$-type diffused regions, the Fe will probably either form $\mathrm{FeB}$ pairs or interstitial Fe (although mostly the former due to the very small separation between $B$ atoms). In either case, the minority carrier capture cross section is much larger ${ }^{11}$-at least $2 \times 10^{-15} \mathrm{~cm}^{2}$. This means the impact of the Fe would be at least a factor of 20 greater in the $p$-type diffusions (note that both centers are effectively "deep" in such heavily doped regions, and so the lifetime simply scales with the minority carrier capture cross section). Here, we have assumed that the Fe in the diffusions remains in pointlike form, avoiding precipitation because of the greatly increased solubility limit for metals in heavily doped regions. However, even if precipitates do form to a significant extent, the fact remains that their presence causes much less recombination in $n$-type emitters than $p$-type.

In general, recombination in emitters with reasonably passivated surfaces is dominated by Auger recombination. For Fe-related centers to have a measurable impact, the recombination rate through them must be at least comparable to the Auger recombination rate. Typical emitter doping concentrations between $10^{19}$ and $10^{20} \mathrm{~cm}^{-3}$ yield low-injection

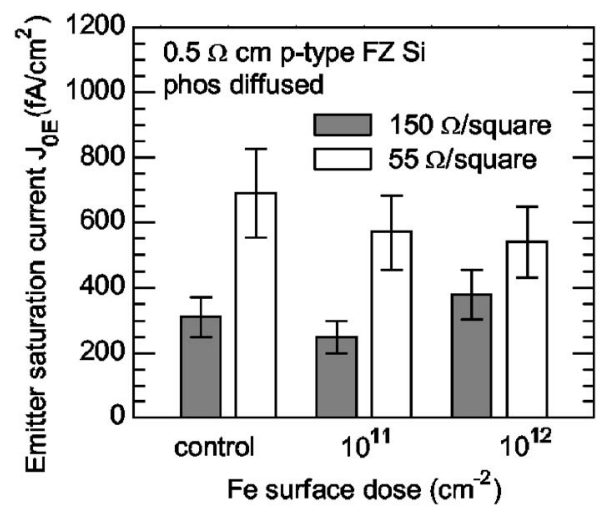

FIG. 3. Emitter saturation currents for the phosphorus diffused samples as a function of Fe dose. 


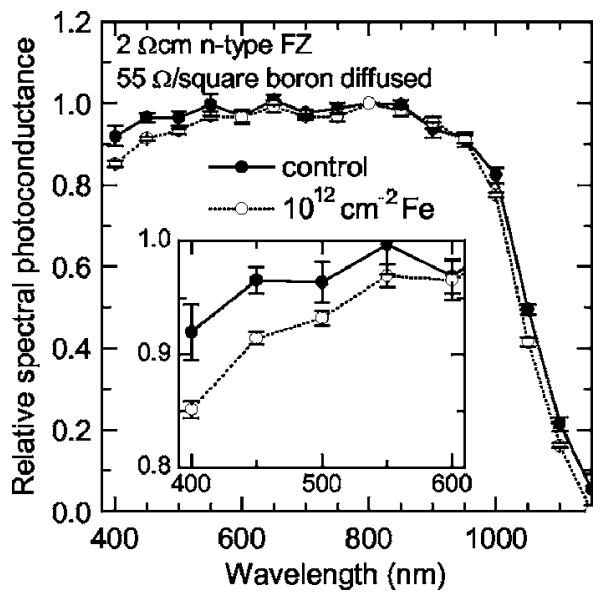

FIG. 4. Relative spectral photoconductance of the control sample and the highest Fe dose sample with heavy boron diffusions.

Auger lifetimes of between $5 \times 10^{-8}$ and $5 \times 10^{-10} \mathrm{~s}$ for $n$-type emitters, and between $10^{-7}$ and $10^{-9} \mathrm{~s}$ for $p$-type emitters. ${ }^{12}$ Fe gettered to the thin emitter regions will have local concentrations of around $10^{16} \mathrm{~cm}^{-3}$ for the heaviest $\mathrm{Fe}$ dose used here (assuming the emitter thickness is three orders of magnitude smaller than the sample thickness). In $n$-type silicon, the corresponding low-injection SRH lifetime for interstitial $\mathrm{Fe}$ would be around $10^{-7} \mathrm{~s}$, higher than the Auger lifetime. On the other hand, in $p$-type emitters, the corresponding lifetime due to $\mathrm{FeB}$ pairs ${ }^{10,11}$ would be approximately between $5 \times 10^{-9}$ and $5 \times 10^{-10} \mathrm{~s}$, and even less for interstitial Fe. These values are comparable to or less than the Auger lifetime, and hence might be expected to impact on the emitter saturation current density of $p$-type emitters.

The increase in recombination in the emitter region in the Fe-containing boron-diffused samples was corroborated by spectrally resolved photoconductance measurements, shown in Fig. 4. The data have been corrected for front surface reflection, giving a quantity that is analogous to the internal quantum efficiency. ${ }^{13}$ The relative photoconductance is reduced in the ultraviolet region for the sample containing Fe. This reflects the fact that electron-hole pairs generated in the emitter are less likely to reach the collecting junction, in qualitative agreement with the $J_{0 E}$ results.

These findings may have some bearing on the production of multicrystalline silicon solar cells. Since current industrial cells have $p$-type bases with $n$-type emitters, gettered Fe will apparently have little impact on emitter recombination. This reflects the desirable outcome of moving Fe from a $p$-type region to an $n$-type region. On the other hand, for $n$-type multicrystalline bases, which are of increasing interest, iron gettered to boron-diffused emitters may still have a significant impact on global recombination. In fact, the $J_{0 E}$ value for the highest Fe dose for the heavy boron diffusion in Fig. 2 corresponds to an implied-open-circuit voltage of $630 \mathrm{mV}$ $\left(25^{\circ} \mathrm{C}\right.$, single-sided diffusion), ${ }^{14}$ reduced from $655 \mathrm{mV}$ for the control. This may prove to be a significant limitation, considering that $p$-type industrial multicrystalline silicon cells achieve voltages approaching $620 \mathrm{mV}$, which also includes all other recombination channels in the base, at the rear surface and in metallized regions. Pregettering any Fe to $n$-type diffused regions, which may anyway be required for rear contacting, would therefore appear to be a good strategy.

In conclusion, we have shown that the presence of moderate levels of $\mathrm{Fe}$ in boron-diffused $p$-type emitters can cause an increase in the emitter saturation current, while phosphorus-diffused $n$-type emitters are immune to its effects. This may be due to the capture cross section asymmetry of the Fe-related centers. In addition, phosphorus diffusions were found to be much more effective at gettering $\mathrm{Fe}$ from the base than boron diffusions.

This work has been supported by the Australian Research Council. The authors acknowledge the assistance of K. Hanton, P. N. K. Deenapanray, S. S. K. Tatineni, R. Ferre, and J. Mitchell, and helpful discussions with P. P Altermatt.

${ }^{1}$ R. A. Sinton, T. Mankad, S. Bowden, and N. Enjalbert, in Proceedings of the 19th European Photovoltaic Solar Energy Conference, Paris (WIP, Munich, 2004), p. 520.

${ }^{2}$ D. Macdonald, A. Cuevas, A. Kinomura, and Y. Nakano, in Proceedings of the 29th IEEE Photovoltaic Specialists Conference, New Orleans, LA (IEEE, New York, 2002), p. 285.

${ }^{3}$ A. A. Istratov, H. Hieslmair, and E. R. Weber, Appl. Phys. A: Mater. Sci. Process. 69, 13 (1999).

${ }^{4}$ R. A. Sinton and A. Cuevas, Appl. Phys. Lett. 69, 2510 (1996).

${ }^{5}$ D. E. Kane and R. M. Swanson, in Proceedings of the 18th IEEE Photovoltaic Specialists Conference, Las Vegas (IEEE, New York, 1985), p. 578.

${ }^{6}$ A. Cuevas, Sol. Energy Mater. Sol. Cells 57, 277 (1999).

${ }^{7}$ D. Macdonald and L. J. Geerligs, Appl. Phys. Lett. 85, 4061 (2004).

${ }^{8}$ P. J. Cousins, D. H. Neuhaus, and J. E. Cotter, Appl. Phys. Lett. 95, 1854 (2004).

${ }^{9} 5$ G. Zoth and W. Bergholz, J. Appl. Phys. 67, 6764 (1990).

${ }^{10}$ D. Macdonald, L. J. Geerligs, and A. Azzizi, J. Appl. Phys. 95, 1021 (2004).

${ }^{11}$ S. Rein, Lifetime Spectroscopy, Vol. 85, Springer Series in Materials Science (Springer, Berlin, 2005).

${ }^{12}$ P. P. Altermatt, J. Schmidt, G. Heiser, and A. G. Aberle, J. Appl. Phys. 82, 4938 (1997).

${ }^{13} \mathrm{H}$. Mäckel and A. Cuevas, in Proceedings of the 20th European Photovoltaic Solar Energy Conference, Barcelona, Spain (WIP, Munich, 2005), p. 1120.

${ }^{14}$ A. Cuevas and R. A. Sinton, Prog. Photovoltaics 5, 79 (1997). 